\title{
Adaptive noise cancellation system elimination of sinusoidal interference*
}

\author{
Li-Min Feng, Wei He ${ }^{\dagger}$, Hua-Fu Li and Xing-Tuan Yang \\ School of Physics and Electronic Information, Yunnan Normal University \\ Kunming, China \\ E-mail:he99wei@aliyun.com
}

\begin{abstract}
Adaptive noise cancellation technology to eliminate background noise is a signal processing technique and a specific application of theoretical processing. Adaptive filter and the corresponding adaptive algorithm is the core component of Adaptive Noise System, With an appropriate algorithm based on adaptive filtering theory for data processing .In the paper, Abandoning the common trap after having some knowledge about adaptive filters and adaptive algorithm ,then using adaptive filter of second order LMS to eliminate sinusoidal interference for different parameters and different signal simulation and using MATLAB sinusoidal to complete the study of Adaptive noise cancellation system eliminates sinusoidal interference.
\end{abstract}

Keywords: Adaptive Noise; Filter; Attenuation; Sinusoidal Interference.

\section{Introduction}

In recent decades, the signal processing technology has made great progress. Wherein the adaptive signal processing usually get in communications, radar, sonar, seismology, mechanical design, navigation systems, biological area, electronics and other widely used [1], it has gradually become an important branch of modern communication process [2]. Adaptive signal processing in communications applications in some of the classic may be main interference cancellation, channel equalization, system identification, signal prediction, adaptive beam forming and so on. Wang Lei, Yang and other key detailed description of the principle of analysis and design process of the filter communications engineering an integral part. Which is also equipped with a circuit diagram of the various filters, amplitude-frequency characteristic diagram, phase frequency characteristic diagram of the filter prototype [3]. This helps to filter understanding. Huang fu Kan, Chen Jian wen, etc. are introduced one by one to the development prospects of the adaptive filter [4]. Model can be used to identify the system model, such as system modeling, estimating system as the

\footnotetext{
* This work is supported by National Natural Science programs (51267021) and College students'
} innovative entrepreneurial training programs (2015) 
adaptive filter characteristic; adaptive antenna, an adaptive filter is used to control the beam direction, and while providing a zero in the beam pattern to eliminate undesirable interference; ECG to eliminate power disturbances, adaptive noise canceller adaptive filter for estimating a desired signal and eliminate the noise component. Jian gong Road and other advantages and disadvantages of the system introduced and the corresponding adaptive filters adaptive algorithm [5], and simulation in MATLAB, and each algorithm has a simulation diagram for easy understanding of the adaptive filter advantages, faster and better application of adaptive filters.

\section{Principle and Calculation}

Filter resonance circuit from the circuit structure, as long as the combination constituting a resonant circuit can be achieved filters, filter all kinds of RF / microwave transmission line formed called distributed parameter filter; theoretically, when lossless filter assembly. As a signal processing system, it is possible to suppress the input signal or the filtered interference signal, extract the useful signal; filter was able to filter, because of its signals at different frequencies have different gains can signal certain frequencies amplification, frequency signals while others are suppressed. The optimal design of the frequency response characteristics and optimal filtering parameters to maximize the filtered interference signal, the optimum filter characteristics and parameters related to the input signal, the optimal filter parameters must be determined according to the characteristics of the input signal. Normal adaptive filter with filter has two important differences: filtering parameters (1) of the adaptive filter is variable, it is possible to change with the external signal to change the parameters of the dynamic characteristics, the optimum filter state holding, the adaptive filter is except ordinary filter hardware as well as software components, namely adaptive algorithm. (2) An adaptive algorithm determines how the adaptive filter according to changes in the external signal to adjust the parameters of the adaptive algorithm directly affects the filtering effect.

The general structure of the adaptive filter shown in Fig.1, $d(n)$ is the desired response, $x(n)$ to the input of the adaptive filter, $y(n)$ is the adaptive filter output, $e(n)$ of the estimation error. Filter coefficients of the adaptive filter by the error signal $e(n)$ control, automatic adjustments $e(n)$ and the value of the adaptive algorithm.

Adaptive (self-adaptive) refers to the processing and analysis process, according to the data processing feature automatically adjusts the data processing method, processing sequence, process parameters, boundary conditions or constraints to the statistical distribution of the data processing, structure features adapted to achieve the best treatment effect. Adaptive process 
mathematical model for the way the process is gradually approaching the target. The mathematical model is adaptive algorithm. Existing adaptive algorithm is divided into categories of LMS and RLS. The LMS algorithm is Widrow and Hoff presented in 1960[6], the algorithm based on the minimum mean square error criterion, based on the gradient method, through the improvement of the mean square error estimation value approach gradient, whichever gradient single error sample as a mean square the estimated value of the gradient. Available Recursive publicity represents the LMS algorithm [7][8].

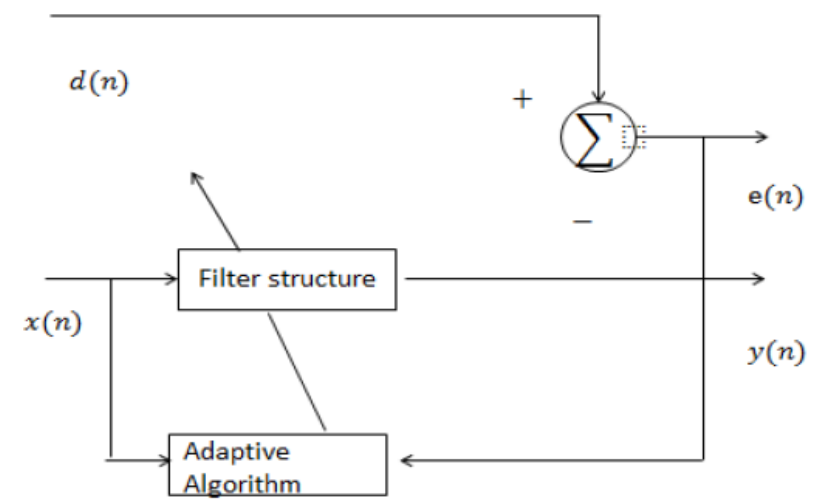

Fig.1. The general structure of the adaptive filter

$$
\begin{gathered}
y(n)=W(n) \cdot X(n) \\
e(n)=d(n)-y(n) \\
W(n+1)=W(n)+2 \mu X(n) e^{*}(n)
\end{gathered}
$$

$W(n)$ is the filter coefficient vector, the weight vector may be viewed as filter coefficients; $X(n)$ is the input signal from the group consisting of an input vector; $y(n)$ is the input signal, $d(n)$ of the desired signal ; $e(n)$ is an error signal; step factor $\mu$ is the weight vector update, $\mu$ greater the faster the convergence, but after the error signal greater convergence, $\mu$ is smaller, the convergence speed more slow, error signal after convergence is also smaller, so you can tune the performance of LMS algorithm by adjusting the step factor $\mu$ value.

\section{Simulation and Conclusions}

Traditional broadband signal sinusoidal interference suppression method is to use notch filter (notch filter), for which we need to know the precise sinusoidal 
interference signal frequency, however, when the interference is slowly varying sinusoidal frequency. And frequency selection feature requires very sharp, then the best use of adaptive noise cancellation method. Below is a program of a second order LMS adaptive filter to eliminate sinusoidal interference.

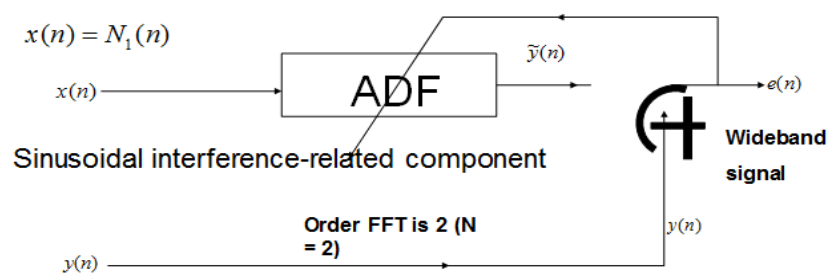

Fig. 2. A second order LMS adaptive filter

$s(n)$ Is white noise is uniformly distributed, $r_{0}=0.05$. Wideband signal sinusoidal interference:

$$
\begin{gathered}
N_{0}(n)=\sin \left(\frac{2 \pi}{16} n+\frac{\pi}{10}\right) \\
N_{0}(n)=\sin \left(\frac{2 \pi}{16} n+\frac{\pi}{10}\right) \\
y(n)=s(n)+N_{0}(n)
\end{gathered}
$$

Equivalence curve plotted with MATLAB errors and error performance curve performance curve as shown in Fig 3.
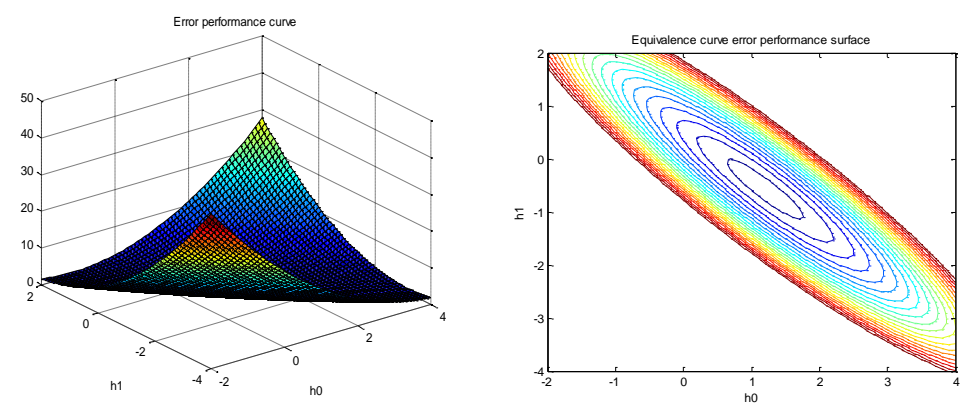

Fig. 3. Error performance curve Equivalence curve error performance surface

Generated by MATLAB variance of 0.05 with zero mean white noise $S(n)$; Its first implementation as shown in Fig 4: 


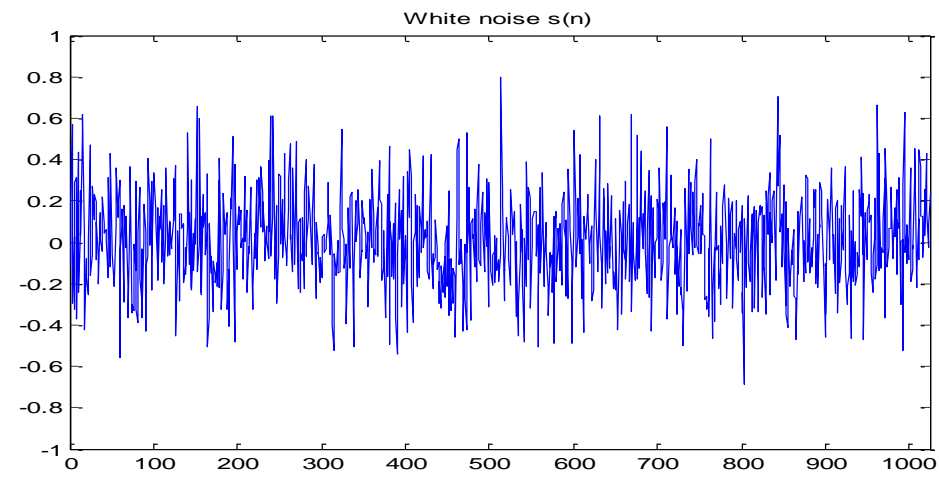

Fig. 4. The first implementation
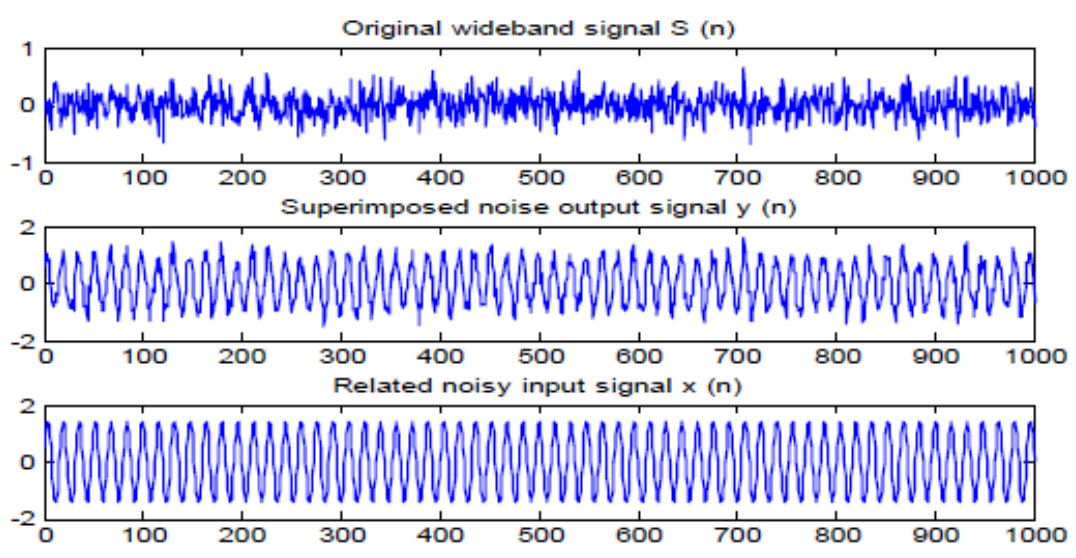

Fig. 5. Steepest descent method and LMS algorithm

Steepest descent method is shown in Fig 5: Steepest descent method iterations trajectory curve as shown in Fig.6(left);Single achieve statistical average (100 experiments at) $J(n)$ and $e(n)$ characteristics, single Realization $J(n)$ and $e(n)$ as shown in Fig.6(right).

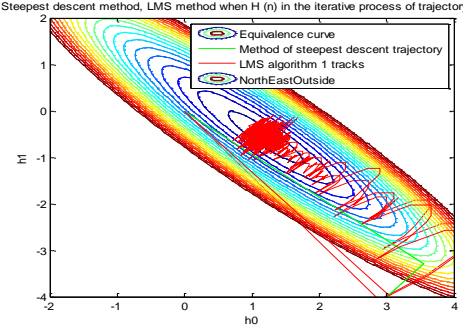

Fig. 6. (left) Single achieve statistical average

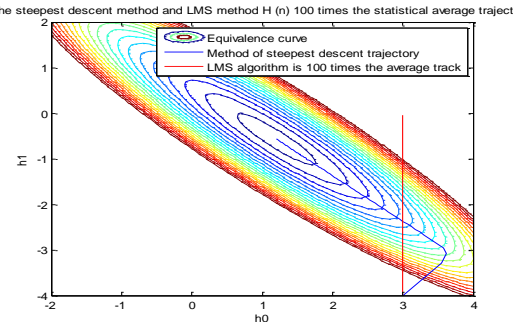

(right) Single Realization $J(n)$ and $e(n)$ 
The simulation result shows that the noise is suppressed, and the input can be quickly converged the feedback, the error signal is reduced greatly.

\section{References}

1. Huangfu Kan, Chen Jian wen, strong green building modern digital signal processing [M]. Beijing: Electronic Industry Press, 2006.

2. (US) Winrow B, Stearns S with, Wang Yongde, Long Hui Xian translation adaptive signal processing [M] Beijing: Mechanical Industry Press, 2008.1: 3.

3. W.P. Ang, B. Farhang-Boroujeny A new class of gradient adaptive step-size LMS algorithms [J] IEEE Trans on Signal Processing, 2001,49 (3): 805-810.

4. Wang Lei, Yang RF circuit design technology [M] Beijing: Electronic Industry Press, 2007.

5. Yong Du, Jian gong Road, Li yuan Zhou digital filter NATALAB and FPGA implementation [M] Beijing: Electronic Industry Press, 2012.3.

6. DU Yong digital filter MATLAB and FPGA implementation [M] Beijing: Electronic Industry Press, 2012.9-62.

7. Du Yong, Lu Jian gong, Li Yuan zhou. MATLAB and FPGA digital filter realization [M]. Beijing: Electronic Industry Press, 2012.3.

8. Simon Hay kin. Zheng Bao yu et al. The principle of adaptive filter [M]. Beijing: Electronic Industry Press, 2010.5. 